\title{
Inadvertent Intrafacet Injection during Lumbar Interlaminar Epidural Steroid Injection: A Comparison of CT Fluoroscopic and Conventional Fluoroscopic Guidance
}

\author{
DP.G. Kranz, (D)A.B. Joshi, (D).A. Roy, (D) K.R. Choudhury, and (D)T.J. Amrhein
}

\begin{abstract}
BACKGROUND AND PURPOSE: Inadvertent intrafacet injection can occur during interlaminar epidural steroid injection, resulting in a false-positive loss of resistance and nontarget injection of medication. The purpose of this investigation was to compare the observed rates of this phenomenon during lumbar interlaminar epidural steroid injection performed by using conventional fluoroscopic and CT fluoroscopic guidance.
\end{abstract}

MATERIALS AND METHODS: We retrospectively reviewed 349 lumbar interlaminar epidural steroid injections performed by using conventional fluoroscopy or CT fluoroscopic guidance to determine the observed rates of inadvertent intrafacet injection with each technique. Cases of inadvertent intrafacet injection were classified as either recognized or unrecognized by the proceduralist at the time of the procedure. Multivariate logistic regression was used to determine the independent effect of imaging guidance technique, age, and sex.

RESULTS: The rate of inadvertent intrafacet injection was observed to be $7.5 \%$ in the CT fluoroscopic group and $0.75 \%$ in the conventional fluoroscopy group. All 16 cases identified from CT fluoroscopic procedures were recognized during the procedure; the single case identified from conventional fluoroscopy procedures was not recognized prospectively. The type of imaging guidance showed a statistically significant effect on the detection of the phenomenon (OR for conventional fluoroscopy versus CT fluoroscopy $=0.10, P=.03$ ) that was independent of differences in age or sex.

CONCLUSIONS: Inadvertent intrafacet injection is identified during CT fluoroscopic-guided interlaminar epidural steroid injection at a rate that is 10 -fold greater than the same procedure performed under conventional fluoroscopy guidance.

ABBREVIATIONS: $C F=$ conventional fluoroscopy; $C T F=C T$ fluoroscopy; ILESI = interlaminar epidural steroid injection

nadvertent intrafacet injection during interlaminar epidural steroid injection (ILESI) can result in nontarget injection outside the epidural space. ${ }^{1}$ It is thought to be due to unintentional needle entry into the retrodural space of Okada, a space located dorsal to the ligamentum flavum that allows communication between the bilateral facet joints and interspinous bursa. ${ }^{2,3}$ Needle placement into this space causes a false-positive loss of resistance, which can mimic the loss of resistance normally felt during entrance into the epidural space.

The observed incidence of inadvertent intrafacet injection during attempted ILESI by using fluoroscopic guidance has been

Received May 27, 2016; accepted after revision September 2.

From the Departments of Radiology (P.G.K., K.R.C., T.J.A.), Orthopedic Surgery (A.B.J.), and Anesthesiology (L.A.R.), Duke University Medical Center, Durham, North Carolina.

Please address correspondence to Peter G. Kranz, MD, Department of Radiology, Duke University Medical Center, Box 3808, Durham, NC 27710; e-mail: peter.kranz@ duke.edu; @PeterGKranz

http://dx.doi.org/10.3174/ajnr.A5000 previously reported to be $1.2 \% .{ }^{4}$ However, most cases (63\%) are not recognized by the operator at the time of the injection. ${ }^{4}$ Thus, most instances of this type of injection result in nontarget delivery of medication and, as a result, do not successfully treat the intended pathology. Although the observed incidence of this phenomenon has been studied by using fluoroscopic guidance, the rate of occurrence during CT fluoroscopy (CTF)-guided injections, which may be more sensitive to detection of small amounts of intrafacet contrast, has not yet been studied, to our knowledge.

The purpose of this investigation was to determine the observed rate of inadvertent intrafacet injection resulting in falsepositive loss of resistance during CTF-guided ILESI and to compare that with the rate observed during conventional fluoroscopy (CF)- guided ILESI at a single institution.

\section{MATERIALS AND METHODS}

This study is a retrospective investigation of lumbar ILESIs performed under CTF or CF guidance at a single institution. Cases 

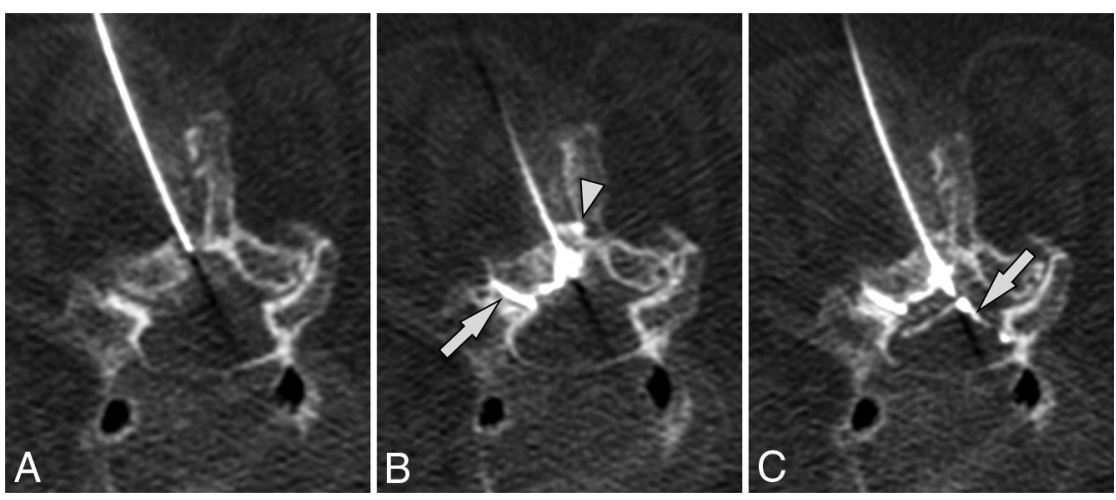

FIG 1. Inadvertent intrafacet injection in an 84-year-old woman with back pain who underwent ILESI with CTF. A, Preinjection axial CTF image before needle placement into the ligamentum flavum. B, Initial CTF image obtained after loss of resistance shows contrast in the facet joint ipsilateral to the injection (arrow). Contrast also pools around the needle tip in the retrodural space of Okada and extends into the interspinous bursa (arrowhead). C, The needle was subsequently advanced through the ligamentum flavum. Contrast is now seen in the epidural space (arrow).

were identified by using the institutional electronic medical record to review procedure schedules. For the CTF-guided cases, all consecutive outpatient lumbar ILESIs performed by a single proceduralist during an 18-month period were included. For the CF-guided cases, all consecutive outpatient lumbar ILESIs performed by 7 proceduralists during an 18-month period were included. The start time for collection of CF-guided cases was 17 months later than that for CTF-guided cases because of differences in when CF-guided cases were archived in a unified institutional PACS. The exclusion criteria were technically inadequate intraprocedural images or images that were missing from the institutional PACS.

The proceduralist performing the CTF-guided ILESIs was a board-certified radiologist with a Certificate of Added Qualification in neuroradiology with 8 years' experience performing spine interventions under CTF guidance. The CF-guided injections were performed by 7 proceduralists ( 1 board-certified physiatrist and 6 board-certified anesthesiologists) with 2-29 years' experience performing spine interventions under fluoroscopic guidance. The average number of years of proceduralist experience per injection in the CF cohort was 7.7 years.

Demographic data and procedural reports were obtained from the electronic medical record. The study was approved by our local institutional review board and was compliant with Health Insurance Portability and Accountability Act regulations.

\section{Injection Technique}

CTF-guided procedures were performed by using a posterior oblique interlaminar approach, as previously described. ${ }^{5}$ Briefly, a 22-ga Quincke-Point Needle (Becton-Dickinson, Washington, DC) was placed into the ligamentum flavum by using intermittent CTF, a syringe containing contrast (iopamidol, Isovue-M 200; Bracco, Princeton, New Jersey) was attached, and the needle was then advanced until loss of resistance was obtained. The volume of contrast injected was typically approximately $0.5 \mathrm{~mL}$. Immediately on loss of resistance, an additional image was obtained to evaluate the location of the injected contrast. All images from the procedure were archived in the PACS.

\section{Image Analysis}

\section{Statistical Analysis} or $\mathrm{CF}$ ), age, and sex.
CF-guided injections were performed by using a method similar to that of injections performed under CTF. ${ }^{6}$ However, an 18- or 20-gauge Tuohy needle was used, and the epidurogram was obtained under either live or spot fluoroscopy, according to operator preference. Some proceduralists in the CF group used saline to judge loss of resistance; location was subsequently confirmed by all proceduralists with approximately $2 \mathrm{~mL}$ of contrast (Isovue-M 300). All proceduralists used at least 2 views, an anteroposterior view and either a lateral or $55^{\circ}$ contralateral oblique view. The fluoroscopic images documenting the injected contrast and final needle position were archived in the PACS.

Intraprocedural images from both CTF- and CF-guided procedures were retrospectively reviewed on the PACS to determine whether inadvertent contrast injection into the facet joints occurred. A false-positive loss of resistance was determined to have occurred if contrast medium was documented in the facet joint before injection of contrast into the epidural space (Fig 1). Cases of inadvertent facet injection were classified as either recognized or unrecognized. Cases were considered recognized if images confirmed subsequent advancement of the needle with resultant contrast injection into the epidural space. Cases were considered unrecognized if no further needle adjustment was made.

Initial image review was performed by one of the study proceduralists who performed injections with the same type of imaging technique. For the CTF-guided procedures, cases were reviewed by the performing neuroradiologist. For the CF-guided procedures, cases were reviewed by the study physiatrist. All cases considered either equivocal or positive for inadvertent facet injection were then independently reviewed by a second board-certified radiologist with a Certificate of Added Qualification in neuroradiology who has 5 years' experience performing injections by using both CTF and CF guidance. Cases of disagreement between readers were resolved by consensus.

Initial univariate analysis compared differences in patient ages between groups by using the Mann-Whitney $U$ test, and sex, by using the Fisher exact test. This analysis revealed an unequal age distribution between the CTF and CF groups; multivariate logistic regression was therefore used to examine the independent effect of 3 variables on intrafacet injection: imaging technique (ie, CTF

Statistical analyses were performed by using commercially available software (univariate analysis was performed with GraphPad Prism 6 software, Version 6.0b; GraphPad Software, San Diego, California; multivariate analysis was performed with $\mathrm{R}$ statistical and computing software, Version 3.0.2; http://www.r-project.org/). $P$ values $<.05$ were considered statistically significant. 

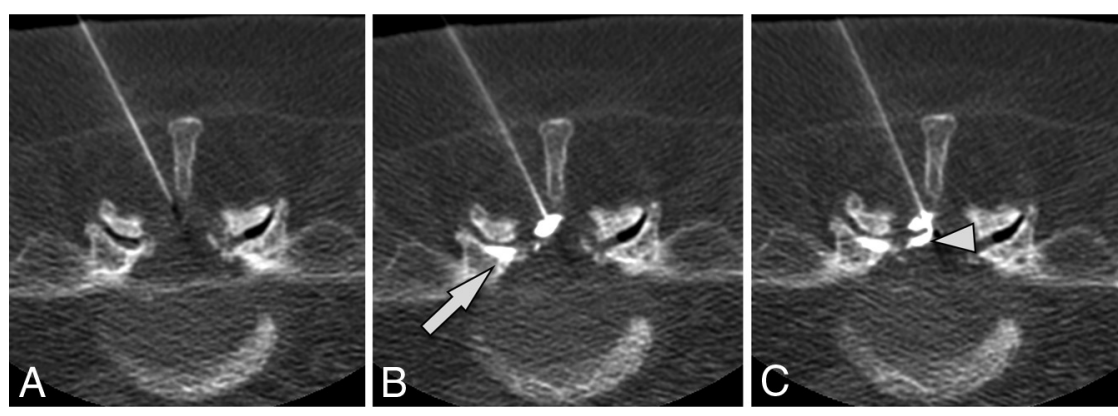

FIG 2. Inadvertent intrafacet injection in a 67-year-old man with spinal stenosis and back pain who underwent ILESI with CTF. A, Preinjection axial CTF image before needle placement into the ligamentum flavum. $B$, Initial CTF image obtained after loss of resistance shows contrast in the facet joint (white arrow). C, Image obtained after needle advancement shows contrast in the epidural space (arrowhead).

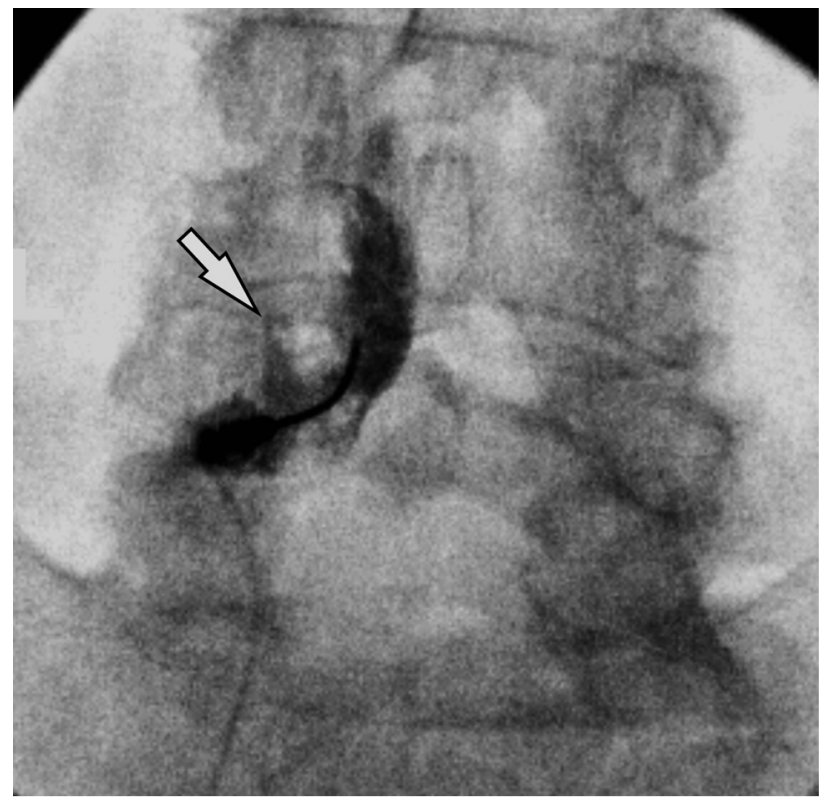

FIG 3. Inadvertent intrafacet injection in a 63-year-old man with a painful L4 -5 central disc protrusion who underwent ILESI by using conventional fluoroscopic guidance. The final procedural image shows a triangular configuration of contrast overlying the inferior aspect of the left L4-5 facet joint, consistent with intrafacet injection.

\section{RESULTS}

A total of 349 lumbar ILESIs were identified. Of these, 214 were performed under CTF-guidance and 135 were performed under CF guidance. No cases were excluded.

The mean subject age for the CTF group was slightly older than the CF group $(65.6 \pm 14.4$ versus $60.6 \pm 13.7$ years $)$, a difference that was statistically significant $(P=.002)$. Female patients composed $56 \%$ of the CTF group and $59 \%$ of the CF group, a difference that was not significant $(P=.51)$.

Sixteen cases of inadvertent intrafacet injection were identified in the CTF cohort, resulting in a rate of $7.5 \%$ (16/214). All 16 cases (100\%) were identified during the procedure, and the needle was repositioned into the epidural space with subsequent technically successful completion of the procedure (Fig 2). All 16 cases identified as positive for inadvertent intrafacet injection by the first reader were also considered positive by the second reader.

In the CF cohort, 1 case of inadvertent facet injection was identified (Fig 3), resulting in an overall rate of $0.75 \%(1 / 135)$. In this case, the proceduralist did not recognize the inadvertent injection during the procedure. This case was considered equivocal by the first reader, positive for inadvertent intrafacet injection by the second reader, and positive on consensus read. No disagreement between readers occurred in the remaining $\mathrm{CTF}$ or $\mathrm{CF}$ cases.

Because of the unequal age distribution between the CTF and CF groups, multivariate logistic regression was used to determine the independent effect of 3 variables on the rate of recognition of intrafacet injection: type of image guidance, age, and sex. Of these 3 factors, the only variable that showed a statistically significant effect was the type of image guidance: The CF group showed a significantly lower rate of recognized intrafacet injections compared with the CTF group (OR for $\mathrm{CF}$ versus $\mathrm{CTF}=0.10 ; 95 \% \mathrm{CI}, 0.01-0.80 ; P=.03$ ). Increasing age was associated with a nonsignificant trend toward a small increase in risk $(\mathrm{OR}=1.04, P=.06)$. Sex was not significantly associated with the risk of intrafacet injection $(P=.09)$.

Of the 17 cases in which intrafacet injection was identified, all (100\%) had contrast flow into the facet ipsilateral to the injection. Additional contrast flow into the contralateral facet joint was seen in 2 cases (12\%) and into the interspinous bursa in 3 cases (18\%). Injections were distributed along the entire lumbar spine, with cases positive for intrafacet injection seen at L1-2 $(n=1)$, L3-4 $(n=7), \mathrm{L} 4-5(n=8)$, and L5-S1 $(n=1)$. Of the cases performed under CTF guidance, a widened facet joint $(>2 \mathrm{~mm})$, with or without gas in the joint, was noted at the injected level in 5 cases (31\%).

\section{DISCUSSION}

Identification of inadvertent intrafacet injection during attempted lumbar ILESI is important because it results in the falsepositive loss of resistance and, if not recognized, may decrease the effectiveness of the procedure due to nontarget delivery of medication. Previous studies of CF-guided ILESI have demonstrated that these injections are often overlooked at the time of the procedure and that the contrast pattern associated with such injections may mimic a true epidural injection. ${ }^{1,4}$ Our investigation showed that inadvertent intrafacet injection was identified during $7.5 \%$ of CTF-guided lumbar ILESIs, a rate 10 -fold higher than the rate observed during CF-guided injections. The rate of $0.75 \%$ we observed for CF-guided injections is generally comparable with the rate of $1.2 \%$ previously reported in the literature for CFguided procedures. ${ }^{4}$

Previous anatomic investigations have identified mechanisms for the communication between the interlaminar space and the facet joint. In 1981, Okada ${ }^{7}$ described an anatomic space located dorsal to the ligamentum flavum that allows communication between the bilateral facet joints and the interspinous region at a single spinal level. This space has become known as the "retrodural space of Okada" and may be identified on imaging during 
spinal procedures in which contrast is injected or in cases in which infection spreads along this space. ${ }^{2,8}$ Spread of contrast in the retrodural space of Okada specifically during lumbar ILESI, resulting in intrafacet injection, has been previously documented in the literature. ${ }^{3}$ In a postmortem anatomic study, Xu et $\mathrm{al}^{9}$ showed that in some patients, the facet joint capsule extends not only dorsal to the ligamentum flavum but also into the ligamentum flavum itself. This relationship would provide a pathway for intrafacet spread of contrast (and resultant loss of resistance) while a needle passes through the ligamentum flavum before entry into the epidural space.

There are 2 possible explanations for the difference in rates of identified inadvertent intrafacet injections between CTF and CF: The phenomenon may be more common during CTF-guided procedures or it may be more readily recognized during CTFguided procedures.

For the first explanation to be true, there would need to be procedural differences between CTF- and CF-guided ILESI that could account for this difference. The techniques for performing the ILESI under fluoroscopic guidance and CTF-guidance are generally the same; however, in our investigation, the proceduralists used different needle types (Tuohy for CF versus QuinckePoint Needle for CTF). This difference might have had an effect on the observed rates of intrafacet injection. Other procedural variables such as the spinal level of injection and relative laterality of needle placement within the interlaminar space would not be likely to account for the difference because they have been previously investigated and shown not to affect the likelihood of inadvertent intrafacet injection. ${ }^{1,4}$

An alternate explanation is that the cross-sectional nature of the imaging used with CTF is more sensitive for the detection of this type of injection. Fluoroscopic imaging would be expected to be less likely to detect inadvertent facet injection, and indeed previous evidence shows that most cases occurring during CF-guided procedures go unrecognized. In a study examining inadvertent intrafacet injections during 686 lumbar ILESIs performed under CF guidance, only $37.5 \%$ of inadvertent intrafacet injections were prospectively identified by the proceduralist at the time of the original procedure. ${ }^{4}$ The same investigators, in a separate report describing the appearance of the phenomenon by using CF, indicated that the imaging appearance of an intrafacet injection might mimic the appearance of a successful epidural injection. They noted that differentiation of intrafacet injection from epidural injection may require injection of a larger volume of contrast sufficient to distend the facet joint capsule, producing more clearly visible contrast in the joint on lateral projection. ${ }^{1}$ This increase in the volume of contrast injection is not commonly performed in routine practice, however. Furthermore, the best projection for recognizing intrafacet injection under fluoroscopy would be an oblique image oriented in the same plane as the facet joint; this view is not routinely obtained during most CF-guided lumbar ILESIs. Given the difficulty in recognizing this injection type, it is certainly plausible that previous estimates of an incidence of $1.2 \%$ during CF-guided procedures may be underestimations.

If the true incidence of intrafacet injection is indeed closer to our estimate of $7.5 \%$ as seen with CTF-guided cases, the clinical impact of this phenomenon would not be trivial. Based on 2011 use data from Medicare alone, more than 914,000 lumbar ILESIs are performed per year in the United States. ${ }^{10}$ Assuming a true rate of $7.5 \%$ for intrafacet injections, the number of attempted ILESIs that resulted in inappropriate intrafacet injection would be in excess of 68,500 per year, with most of those cases likely going unrecognized. These cases represent patients for whom the intended epidural injection of steroid would not occur; thus, the target pathology would go untreated.

Elimination of these cases should have the effect of increasing clinical success rates for ILESIs. Of note, all instances of inadvertent intrafacet injection during CTF-guided procedures were prospectively recognized, which would markedly decrease the risk of nontarget injection when this method of imaging guidance is used. Further investigation into how to more reliably detect these injection types during CF-guided ILESI, including the possibility of additional fluoroscopic views to optimize the chances of its visualization, is necessary.

When performing ILESI, one must also remember that intrafacet injection may not be the only potential source of false-positive loss of resistance. False-positive rates of loss of resistance of up to $25 \%-30 \%$ have been reported when using air-filled syringes to enter the epidural space. ${ }^{11,12}$ It is possible that some of these previously reported cases may have been due to unrecognized entry into the retrodural space of Okada. The use of iodinated contrast markedly decreases the rate of incorrect needle-tip placement, ${ }^{13}$ but as mentioned previously, it does not completely protect against error because the appearance of contrast injected into the facet may closely resemble an epidural injection on lateral views. ${ }^{1}$ Proceduralists must take care to confirm that the suspected epidural entry, whether judged by loss of resistance or contrast injection, is confidently confirmed before medication delivery.

Our study has limitations. First, and perhaps most important, this investigation does not definitely establish the reason for the observed difference in rates on intrafacet injection performed under CF and CTF guidance. Although CTF may recognize cases missed under CF, we cannot preclude the possibility that unrecognized differences in the CTF and CF techniques predispose CTF-guided procedures to a higher likelihood of this type of injection. The implementation of conventional fluoroscopic techniques or views that more reliably identify intrafacet injection would help resolve this question by providing a better estimate of the true incidence. Second, the small number of total cases of intrafacet injections could result in failure to detect a statistically significant influence of age and/or sex (ie, a type II error). Age, the closest variable to achieving statistical significance, showed only a very small effect size, however, suggesting that even if there was a statistically significant effect, it would not explain the 10 -fold increase in the rate of intrafacet injection we observed under CTF. Sex was equal between the CF and CTF groups $(P=.51)$ and would therefore not result in the observed difference in rates either.

\section{CONCLUSIONS}

We detected inadvertent intrafacet injection resulting in a falsepositive loss of resistance during CTF-guided lumbar ILESI in 
$7.5 \%$ of procedures, a 10 -fold increase in the rate we observed when the same procedure was performed under conventional fluoroscopy. Because this type of injection can result in nontarget injection of medication if not recognized, further investigation is needed to determine how to optimize detection during CFguided procedures and whether this observed difference in intrafacet injection rates is attributable to differences in technique or whether it is the result of the increased sensitivity of CTF imaging to this type of phenomenon.

Disclosures: Lance A. Roy—UNRELATED: Consultancy: Medtronic, Comments: neuromodulation; Grants/Grants Pending: Pfizer, Comments: drug study; Payment for Lectures including Service on Speakers Bureaus: Medtronic, Comments: neuromodulation.

\section{REFERENCES}

1. Huang AJ, Rosenthal DI, Palmer WE. Inadvertent intra-articular lumbar facet joint injection during fluoroscopically guided interlaminar epidural steroid injection. Skeletal Radiol 2011;40:33-45 CrossRef Medline

2. Lehman VT, Murthy NS, Diehn FE, et al. The posterior ligamentous complex inflammatory syndrome: spread of fluid and inflammation in the retrodural space of Okada. Clin Radiol 2015;70:528-35 CrossRef Medline

3. Murthy NS, Maus TP, Aprill C. The retrodural space of Okada. AJR Am J Roentgenol 2011;196:W784-89 CrossRef Medline

4. Huang AJ, Palmer WE. Incidence of inadvertent intra-articular lumbar facet joint injection during fluoroscopically guided inter- laminar epidural steroid injection. Skeletal Radiol 2012;41:157-62 CrossRef Medline

5. Wagner AL. CT fluoroscopy-guided epidural injections: technique and results. AJNR Am J Neuroradiol 2004;25:1821-23 Medline

6. Bogduk N. International Spine Intervention Society Practice Guidelines for Spinal Diagnostic and Treatment Procedures. San Francisco: International Spine Intervention Society; 2013:409

7. Okada K. Studies on the cervical facet joints using arthrography of the cervical facet joint (author's transl) [Japanese]. Nihon Seikeigeka Gakkai Zasshi 1981;55:563-80 Medline

8. Sarazin L, Chevrot A, Pessis E, et al. Lumbar facet joint arthrography with the posterior approach. Radiographics 1999;19:93-104 CrossRef Medline

9. Xu GL, Haughton VM, Carrera GF. Lumbar facet joint capsule: appearance at MR imaging and CT. Radiology 1990;177:415-20 CrossRef Medline

10. Manchikanti L, Benyamin RM, Hansen H, et al. Reversal of epidural cuts in $\mathbf{2 0 1 5}$ physician payment schedule: two steps forward, one step back. Pain Physician 2014;17:E565-73 Medline

11. White AH, Derby R, Wynne G. Epidural injections for the diagnosis and treatment of low-back pain. Spine (Phila Pa 1976) 1980;5:78-86 CrossRef Medline

12. Bartynski WS, Grahovac SZ, Rothfus WE. Incorrect needle position during lumbar epidural steroid administration: inaccuracy of loss of air pressure resistance and requirement of fluoroscopy and epidurography during needle insertion. AJNR Am J Neuroradiol 2005; 26:502-05 Medline

13. Johnson BA, Schellhas KP, Pollei SR. Epidurography and therapeutic epidural injections: technical considerations and experience with 5334 cases. AJNR Am J Neuroradiol 1999;20:697-705 Medline 